\title{
Educación al paciente en patologías prevalentes y uso de medicamentos en las comunidades de Guano - provincia de Chimborazo
}

\author{
PAULINA VIVANCO ${ }^{*}$, JANETH MONTALVO' \\ ' Facultad de Ciencias Químicas de la Universidad Central del Ecuador \\ *Correspondencia: paulyviv@hotmail.com
}

Recibido: 04 febrero 2011 / Aceptado: 30 abril 2012

\section{Resumen}

La educación al paciente, dándole la información adecuada sobre la prevención de enfermedades, manipulación y uso racional de medicamentos, es una de las herramientas principales, para combatir los malos hábitos alimenticios, de higiene y automedicación, que son prácticas habituales en las comunidades indígenas de la provincia de Chimborazo-Ecuador.

En este estudio, se realizaron encuestas de diagnóstico sobre los problemas anteriormente expuestos, con el fin de conocer cuáles deberían ser los temas idóneos a tratar en las comunidades escogidas para el proyecto. Luego se estructuró un temario sobre clases de enfermedades prevalentes tales como: Parasitosis, Diarrea, Desnutrición, Infecciones Respiratorias y Dermatitis Alérgica; así como también, sobre asuntos relacionados con medicamentos: concepto básico de medicamento, formas farmacéuticas, manipulación adecuada de medicamentos y uso racional de medicamentos; temáticas que fueron dictadas durante tres meses en dichas comunidades. Dicho temario fue desarrollado de manera paulatina cada semana, e incluía, evaluaciones orales para los padres y madres de familia, y evaluaciones escritas para los infantes.

Posteriormente, cuando se culminó el programa de actividades, se realizó una encuesta final para evaluar, de manera global, los conocimientos adquiridos y el impacto alcanzado en las comunidades con el trabajo realizado.

En el presente proyecto, se trabajó con niños y niñas de 4 a 7 años de edad y con los padres-madres de familia de los mismos, con la finalidad de que el aprendizaje sobre las enfermedades prevalentes de la zona y la manipulación adecuada de medicamentos, sea completo y lo pongan en práctica fácilmente en sus hogares.

La aplicación de este proyecto tuvo resultados positivos y significativos en cuanto al cambio de los malos hábitos de vida y a la inadecuada manipulación de medicamentos por parte de los indígenas pertenecientes a las comunidades donde se trabajó; logrando de ese modo, el objetivo principal de esta tesis, que es educar sobre la prevención de las enfermedades prevalentes de la zona.

Palabras clave: Pueblo indígena, patología, medicamento, enfermedad prevalente.

\section{Patient education in prevalent diseases and medication use in communities Guano - Chimborazo province}

\section{Abstract}

Patient education, giving appropriate information on disease prevention, handling and rational use of drugs, is one of the main tools to combat unhealthy eating habits, hygiene and self-medication, which are common practices in indigenous communities Chimborazo province. 
In this study, we conducted diagnostic surveys, about the matters stated above, in order to know, what should be appropriate to address issues in the communities chosen for the project, then a syllabus is structured classes on prevalent diseases such as the parasites, diarrhea, malnutrition, respiratory infections and allergic dermatitis, and drug-related issues such as: basic concept of medication, dosage forms, proper handling of drugs, Rational Use of Drugs, which were issued for three months in communities.

This agenda was developed gradually each week, in which oral assessments were parents, and written for children.

Later, when he completed the program of activities, we conducted a final survey to assess comprehensively, foreground, and the impact achieved in communities with their work.

In this project, we worked with children from 4 to 7 years old and parents of the same, with the aim of learning about the diseases prevalent in the area and the proper handling of drugs is complete and easily implement in their homes.

The implementation of this project had significant positive results in the change of bad habits, and improper handling of medicines by the Indians from communities in which they worked, thus achieving the main goal this thesis, which was to educate on the prevention of diseases prevalent in the area.

Key words: Indigenous pathology, medication prevalent disease.

\section{Introducción}

Las comunidades indígenas del país viven en condiciones precarias, esto las hace susceptibles a un sinnúmero de patologías leves (digestivas, respiratorias, etc.), pero que se vuelven casos graves o hasta crónicos, cuando no han sido tratados de la manera adecuada y no han recibido atención médica oportuna.

La población de estos lugares, al no tener los recursos económicos suficientes para acceder a una atención médica digna, o en su defecto, a medicamentos con la prescripción adecuada, muchas veces muere por complicaciones de enfermedades que no fueron tratadas a tiempo y apropiadamente.

Al ser muy amplio el número de comunidades afectadas, se tomaron como muestra, para la realización del presente proyecto, tres localidades que son: Santa Rosa, San Rafael y Santa Lucía de Chuquipogyo, pertenecientes al cantón Guano, provincia de Chimborazo.

Los/as indígenas, por vivir en zonas muy alejadas de las grandes ciudades donde no poseen los servicios básicos, corren mayores riesgos de afecciones respi- ratorias y estomacales, en su mayoría, por falta de educación con respecto a su forma de vida de condiciones poco higiénicas, ya que no cuentan con agua potable; y por otro lado, por la escasa información que tienen acerca de la manipulación de los medicamentos que son prescritos por los médicos de los subcentros de salud zonales, para aliviar sus enfermedades, dichos centros a los cuales esta gente accede con muy poca frecuencia, por la lejanía existente entre éstos y sus comunidades.

\section{Metodología}

- Una vez recopilada la información, suministrada por la Dirección Provincial de Salud de Chimborazo (DPSCH) y Puesto de Salud de Tuntatacto, se determinaron las 5 patologías prevalentes en las tres comunidades escogidas.

- El diagnóstico de la problemática sobre salud y medicamentos en las respectivas comunidades indígenas, se realizó mediante encuestas a la población de dichas comunidades, con un cuestionario que 
refleja, la percepción que la gente tiene respecto a las enfermedades que padecen, y cuál es la manipulación que les dan a los medicamentos cuando pueden acceder a ellos.

- La organización de las campañas de educación al paciente en las comunidades indígenas anteriormente citadas, se realizaron con los directores de las escuelitas comunitarias y sus profesores/as, con quienes se acordó trabajar, los días miércoles, con la Comunidad de Santa Lucía, los jueves, con la comunidad de Santa Rosa, y los días viernes, con la comunidad de San Rafael de Chuquipoggyo; además, se acordó que dentro del mismo día, se realizarían las clases para los adultos y los niños, con una duración de dos horas cada clase, una vez por semana, durante un tiempo de tres meses.

- Luego de la culminación del programa de las clases impartidas, se elaboró una hoja a manera de encuesta, para evaluar el impacto alcanzado y el nivel de conocimiento adquirido. Las encuestas fueron realizadas una semana después de la última clase dictada, de forma individual a cada participante y en los mismos horarios. Cada clase ejecutada, se llevó de manera organizada mediante fichas de Planificación Didáctica.

- Por pedido de las madres y padres de familia, se entregaron los boletines informativos, al finalizar los tres meses del curso, en una carpeta ordenada de manera ascendente (clase por clase) como respaldo del aprendizaje adquirido.

- La asistencia de los miembros de las diferentes comunidades indígenas, que se encuentran dentro de este proyecto, se consignó en un formato único realizado por la tesista.

\section{Resultados $y$ análisis}

La Encuesta de Impacto se la aplicó a 72 niños de educación inicial, primero, segundo y tercero de educación básica, y a 61 padres y madres de familia.

\section{Análisis de resultados de la encuesta de impacto en los niños/as}

Interpretación de los resultados: De los 72 niños encuestados/as, el 95\% considera que han aprendido

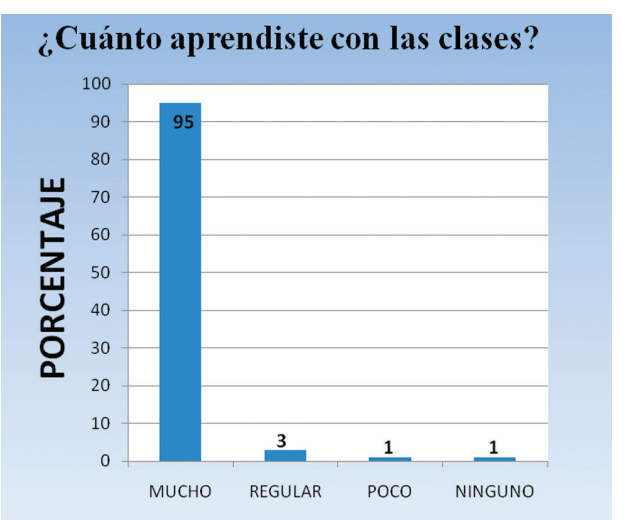

mucho con la capacitación recibida; el 3\% indica que su aprendizaje ha sido regular; mientras un $1 \%$ aprendió poco, y otro $1 \%$ restante, no se acogió a ninguna de las respuestas.

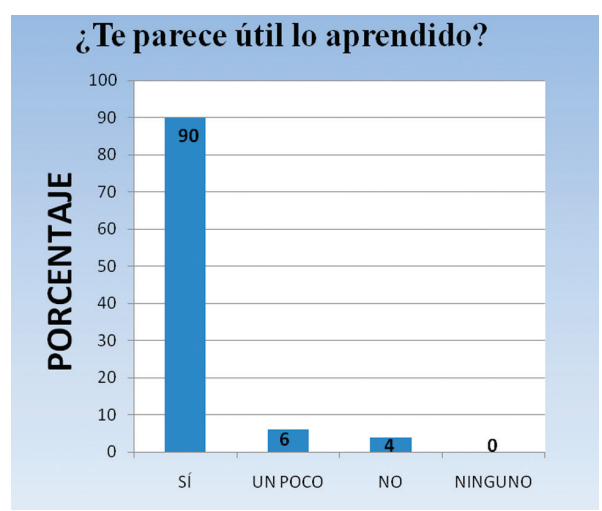

Interpretación de los resultados: De los 72 niños encuestados/as, el $90 \%$ considera útiles los conocimientos que adquirieron; el $6 \%$ indica que fue poco útil lo que aprendieron; mientras que el $4 \%$ considera inútiles los conocimientos adquiridos. Ningún infante se acogió a la última opción.

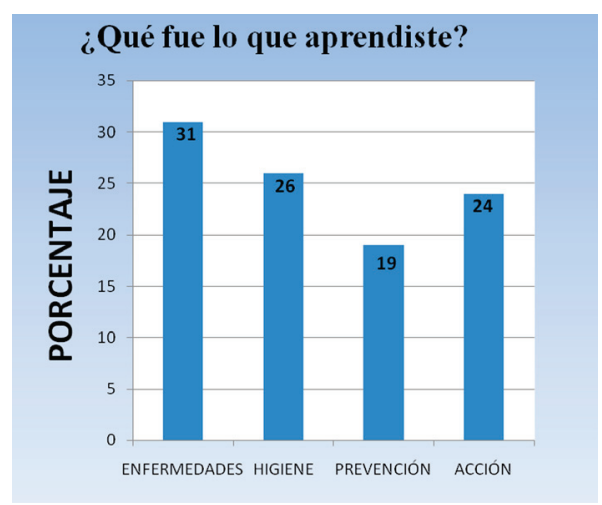

Interpretación de los resultados: De los 72 niños encuestados/as, al $31 \%$ le impactó más las clases sobre las enfermedades; el $26 \%$ aprendió más acerca de las clases de higiene; el 19\% aprendió sobre los métodos de prevención de enfermedades, y al $24 \%$ le impactó 
más lo que deben hacer cuando tienen una enfermedad.

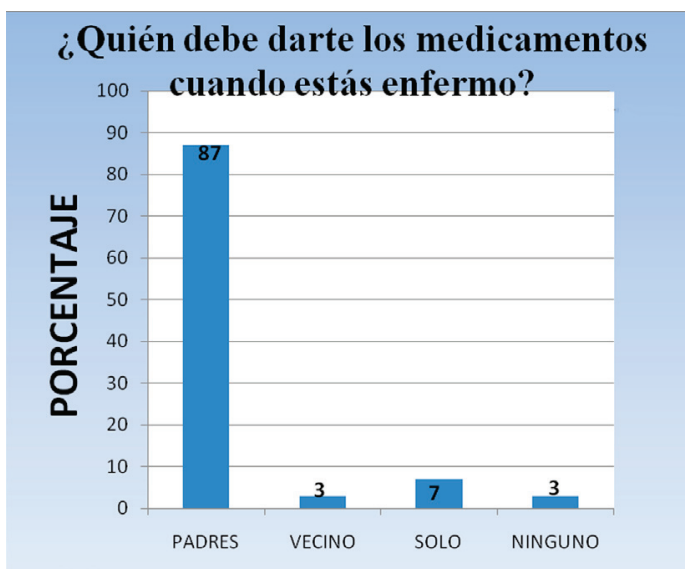

Interpretación de los resultados: De los 72 niños encuestados/as, el $87 \%$ comentó que sus padres-madres son quienes les proveen los medicamentos cuando se enferman; el $3 \%$ cree que sus vecinos/as pueden administrarles los medicamentos; el $5 \%$ considera que se encuentran capacitados para administrarse solos los medicamentos; y el 3\% no se acogió a ninguna de las respuestas anteriores.

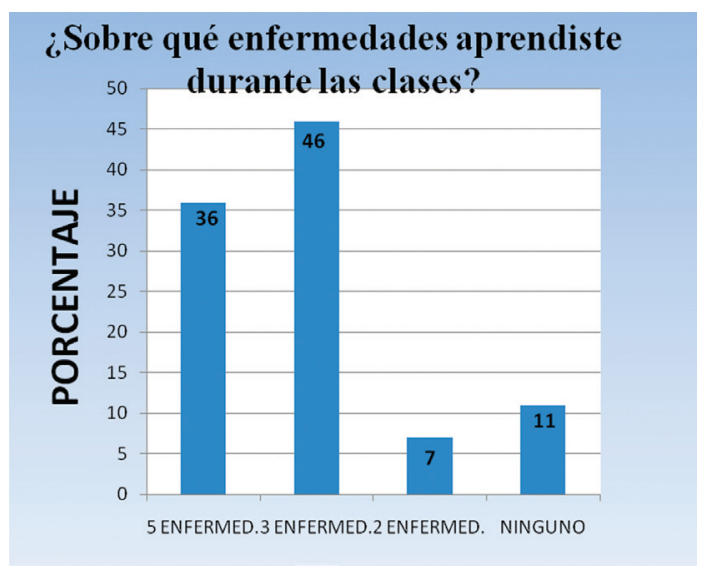

Interpretación de los resultados: El 36\% tiene claros sus conocimientos, luego de las capacitaciones, respecto a las cinco enfermedades prevalentes (infecciones respiratorias, diarrea, parasitosis, desnutrición, e infecciones de la piel); el $46 \%$ tiene claro el conocimiento adquirido sobre tres de las enfermedades prevalentes (infecciones respiratorias, diarrea y parasitosis), dando como resultado una respuesta regular; el $7 \%$ dieron una respuesta insuficiente, ya que solo tenían conocimiento claro de dos de las enfermedades prevalentes, mientras el $11 \%$ no escogió ninguna de las respuestas anteriores, dando como resultado una respuesta incorrecta.

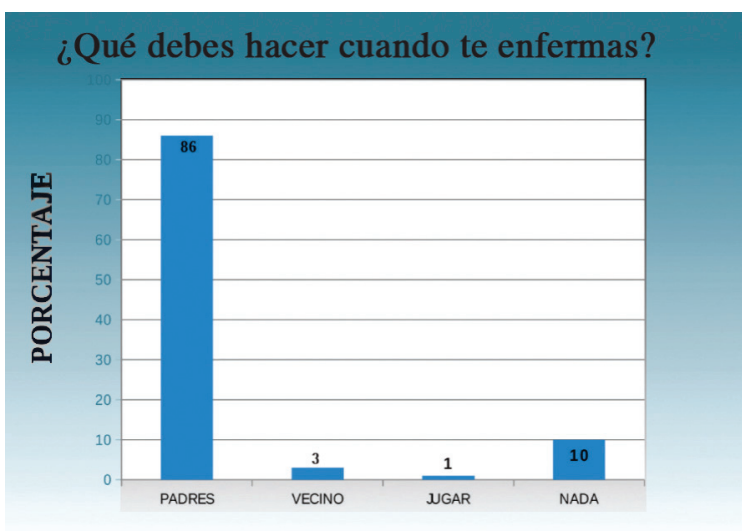

Interpretación de los resultados: De los 72 niños encuestados/as, el $86 \%$ considera necesario avisarles a sus padres cuando se sienten enfermos; el $3 \%$ piensa que deben avisarles a uno de sus vecinos; el $1 \%$ opina que deben jugar cuando están enfermos, y el 10\% piensa que no deben hacer nada cuando se encuentran enfermos.

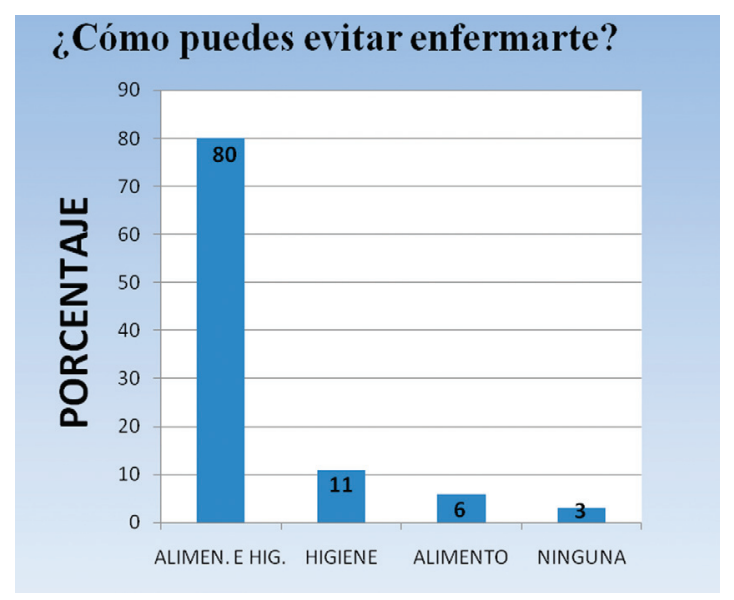

Interpretación de los resultados: De los 72 niños encuestados/as, el $80 \%$ dio la respuesta correcta (buena alimentación e higiene); un $11 \%$, opina que solo es necesaria una buena higiene para prevenir enfermedades; el $6 \%$ dice que solo una buena alimentación es necesaria para prevenir enfermedades, y el $3 \%$ no escogió ninguna de las respuestas anteriores, considerándose una respuesta incorrecta.

Interpretación de los resultados: De los 72 niños encuestados/as, el $96 \%$ sabe que, para estar sanos, deben comer frutas, verduras y carnes; el $1 \%$ considera que para no enfermarse debe comer chicles y 


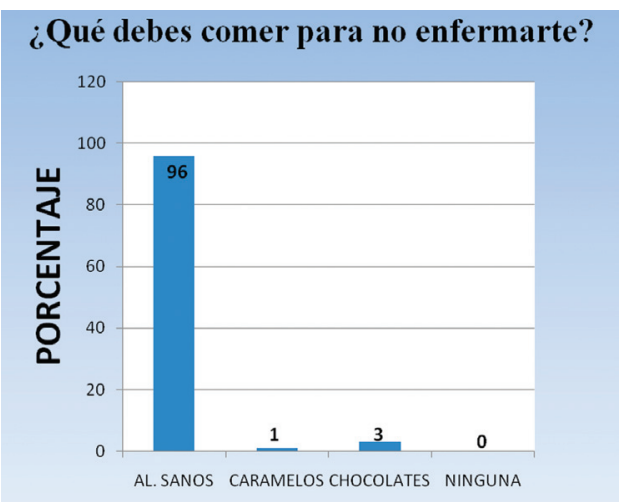

chupetes, y el $3 \%$ considera que para encontrarse en buen estado de salud, debe comer chocolates. Ningún participante escogió la última opción.

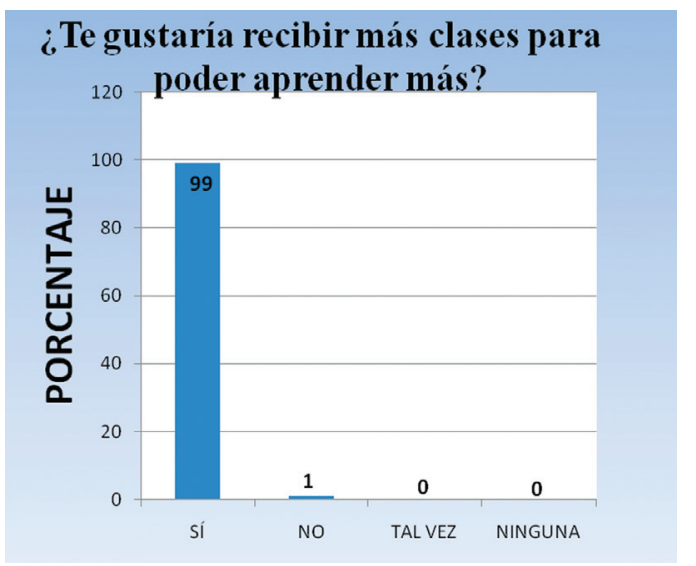

Interpretación de los resultados: De los 72 niños encuestados/as, al 99\% le gustaría recibir más clases acerca del tema para aprender, mientras al $1 \%$ no le interesa saber recibir más respecto al tema. Nadie escogió las dos opciones restantes.

\section{Análisis de resultados de la encuesta de impacto en madres $y / o$ padres de familia}

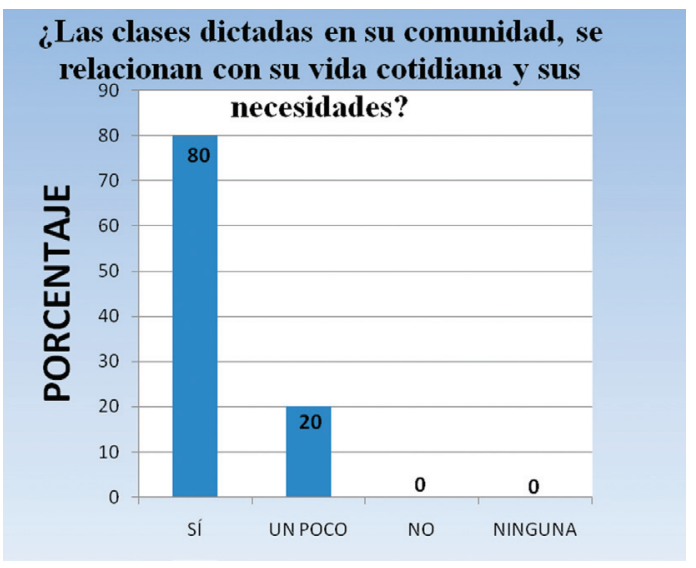

Interpretación de los resultados: De los 61 adultos encuestados/as, el $80 \%$ considera que las clases dic- tadas en su comunidad, se relacionan con su vida cotidiana y sus necesidades, y el $20 \%$ cree que se relacionan un poco, sin existir personas encuestadas que escojan las dos opciones restantes.

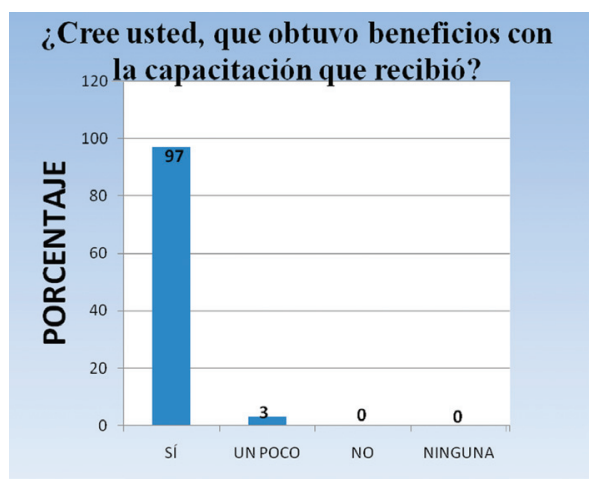

Interpretación de los resultados: De los 61 adultos encuestados, el $97 \%$ cree haber tenido beneficios con la capacitación recibida mientras un 3\% considera que los beneficios son pocos, sin existir personas que escojan las dos últimas opciones.

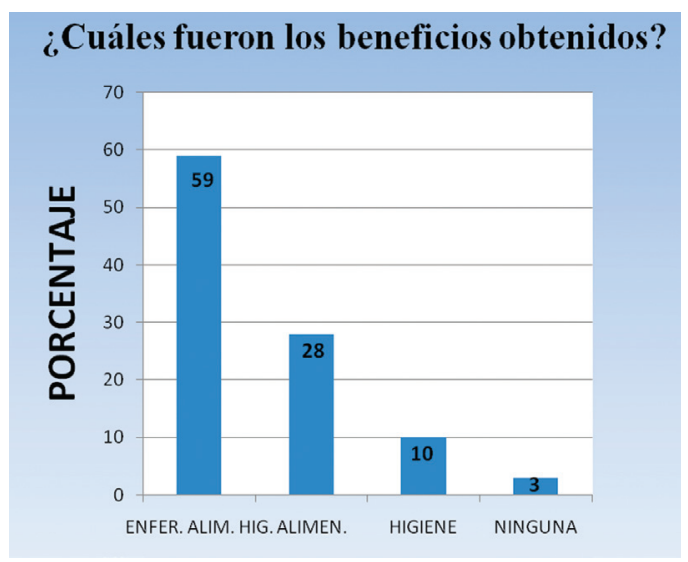

Interpretación de los resultados: De los 61 adultos encuestados/as, el 59\% respondió de forma correcta (aprender sobre enfermedades, importancia de la alimentación e higiene), el $28 \%$ dio una respuesta regular (escogieron dos de las opciones: aprender sobre

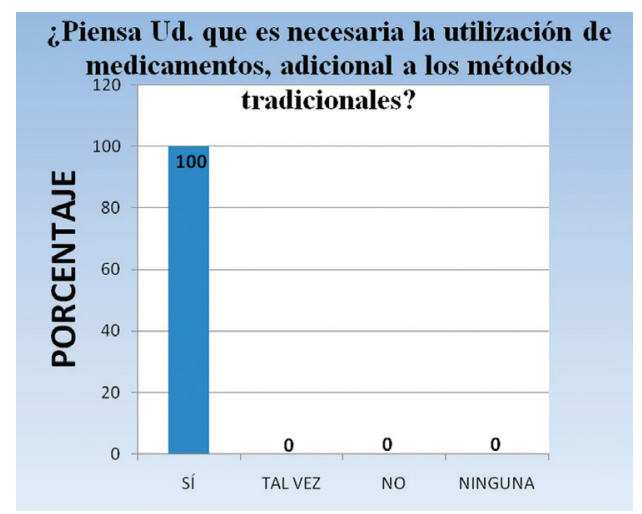


la alimentación e higiene), el 10\% escogió solo una de las respuestas, y el 3\% no escogió ninguna de las respuestas anteriores.

Interpretación de los resultados: De los 61 adultos encuestados/as, el $100 \%$ piensa que es necesaria la utilización de medicamentos para curar enfermedades, adicionalmente a los métodos tradicionales utilizados.

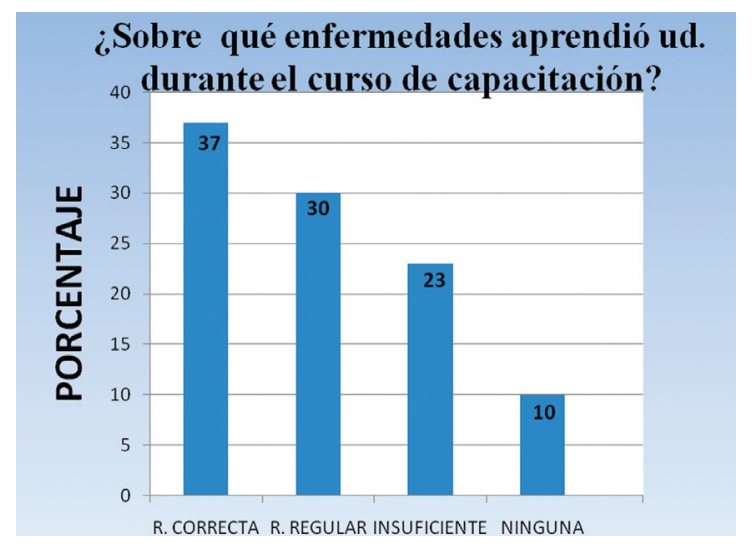

Interpretación de los resultados: De los 61 adultos encuestados, el 37\% dio la respuesta correcta (infecciones respiratorias, diarrea, parasitosis, desnutrición, infecciones de la piel), el 30\% mostró una respuesta regular (solamente nombraron tres de las enfermedades anteriormente mencionadas), el 23\% respondió de forma insuficiente (nombraron una o dos de las enfermedades anteriormente mencionadas), y el 10\% restante, escribió una respuesta incorrecta, al no escoger ninguna de las opciones anteriores.

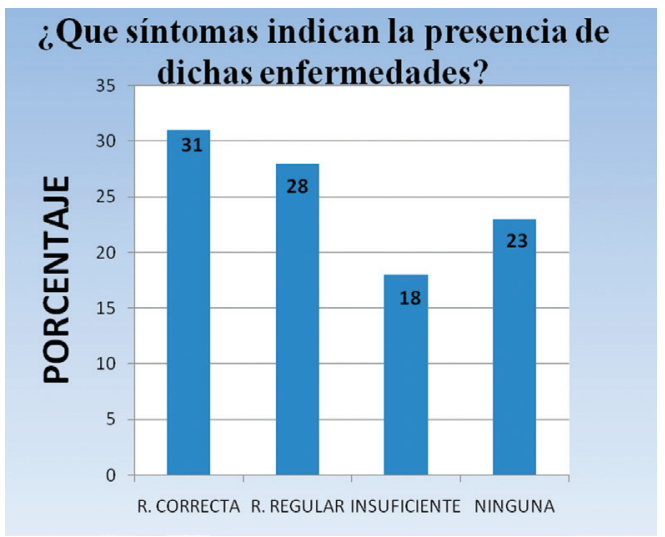

Interpretación de los resultados: De los 61 adultos encuestados/as, el $31 \%$ dio la respuesta correcta (dieron dos síntomas de cada una de las enfermedades analizadas. Para la gripe: tos y dolor de garganta; para la parasitosis: cólicos y niños barrigones; para la diarrea: deposiciones líquidas y frecuentes; para la desnutrición: baja de peso y cansancio; $y$, para las infecciones de la piel: comezón y descamación). Un $28 \%$ dio una respuesta regular (solamente se refirieron a un síntoma de cada enfermedad), el 18\% respondio de forma insuficiente (nombraron indistintamente síntomas de algunas de las enfermedades, sin referirse a todas y de manera no específica), mientras el $23 \%$ restante, dio una respuesta incorrecta, por no escoger ninguna de las opciones anteriores.

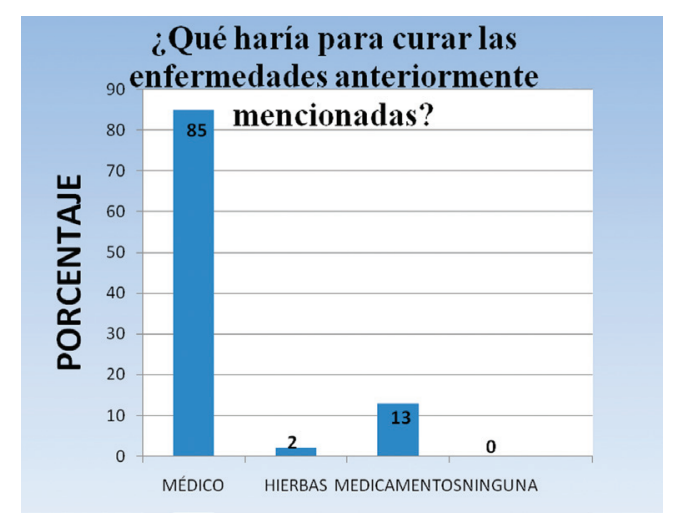

Interpretación de los resultados: De los 61 adultos encuestados/as, el $85 \%$ considera necesario, en caso de enfermedad, acudir inmediatamente al médico/a, el $2 \%$ prefiere seguir con sus métodos tradicionales de curación (infusiones de hierbas y rituales con animales de campo), el 13\% manifiesta que prefieren ir a las farmacias a comprar los medicamentos que les recomienden, y nadie escogió la última opción.

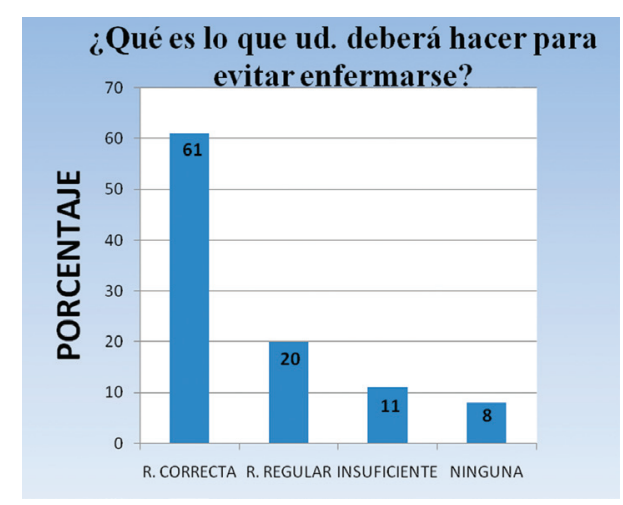

Interpretación de los resultados: De los 61 adultos encuestados/as, el $61 \%$ respondió correctamente la pregunta (buena alimentación, buena higiene personal y del entorno, y protección personal a los factores climáticos), el $20 \%$ dio una respuesta regular (solo nombraron dos de las medidas preventivas anteriormente mencionadas), el $11 \%$ mostró una respuesta insuficien- 
te (solo nombraron una de las medidas preventivas), y el $8 \%$ escribió una respuesta incorrecta, ya que no escogieron ninguna de las opciones anteriores.

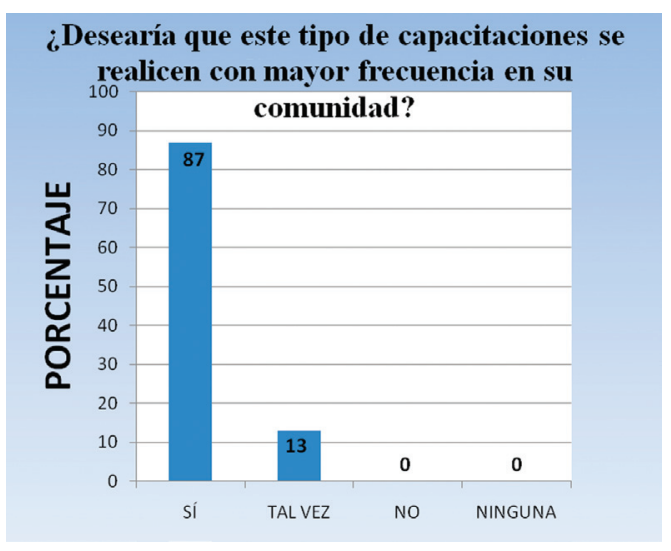

Interpretación de los resultados: De los 61 adultos encuestados/as, el $87 \%$ contesto que sí estarían interesados en que se realicen más capacitaciones de este tipo en su comunidad, el $13 \%$ opinó que tal vez.

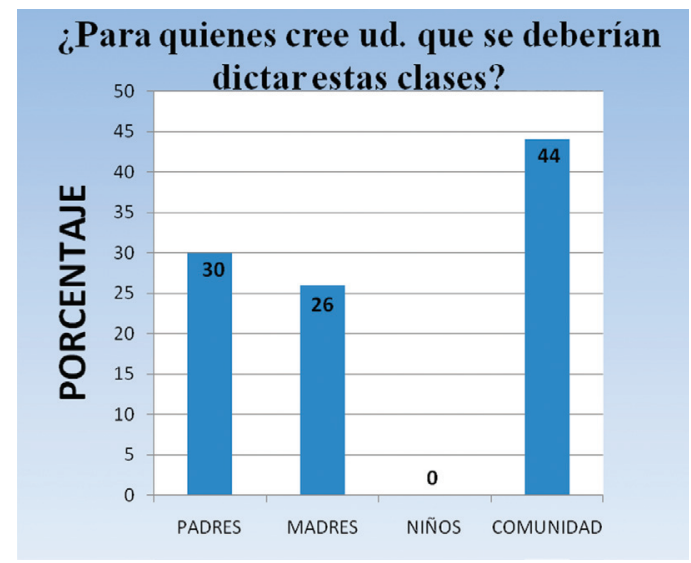

Interpretación de los resultados: De los 61 adultos encuestados/as, el $30 \%$, opina que estas capacitaciones deberían darse a los padres y madres de familia; el $26 \%$ opina que solo a las madres de familia, ninguno de los encuestados/as opina que se deberían dar solo a los niños/as, mientras que el $44 \%$ cree que es necesario que dichas capacitaciones se realicen para toda la comunidad.

\section{Discusión}

- Las madres y/o padres de familia, se encuentran en un número menor al de los alumnos, porque algunos no quisieron de manera voluntaria participar en el proyecto.
- En las comunidades de Santa Rosa y Santa Lucía de Chuquipogyo, existió mucho apoyo y responsabilidad por parte de los integrantes de este proyecto, no siendo así en la comunidad de San Rafael de Chuquipogyo, donde los padres y madres de familia, faltando cuatro semanas para la culminación del proyecto, decidieron no asistir a las capacitaciones, argumentando falta de tiempo, por lo que se retrasó el cronograma de actividades durante dos semanas, recuperando esas clases luego de haber dialogado con los convocados/as.

- Debido a las gestiones realizadas, tanto la Jefatura Política del cantón Guano, como con el Gobierno Parroquial de San Andrés, y dentro de sus posibilidades, ayudaron a las comunidades objeto de estudio, suministrándoles mascarillas para evitar afecciones respiratorias por aspiración de ceniza, debido a la erupción del volcán Tungurahua, y actualmente, están realizando la tramitología necesaria para obtener los recursos y cubrir algunas necesidades de las mencionadas comunidades.

\section{Conclusiones}

- Se logró educar, con la utilización de material didáctico adecuado y de manera efectiva, al 75\% de los adultos y niños de las comunidades en estudio, determinando así un aumento significativo del nivel de conocimientos con respecto a la salud, las enfermedades prevalentes y la importancia de la manipulación y el uso racional de los medicamentos.

- Se determinaron cuáles son las enfermedades prevalentes en cada comunidad, tomando en cuenta los datos recopilados en la DPSCH y el puesto de salud de Tuntatacto, mismos que fueron corroborados por las encuestas de diagnóstico realizadas al inicio del proyecto a todas las personas que participaron en la capacitación.

- Las personas que recibieron la capacitación, aparte de aumentar su nivel educacional, experimentaron una concienciación sobre la salud y lo importante que es prevenir las enfermedades, cambiando algunas costumbres inadecuadas de su estilo de vida (mala alimentación, falta de higiene personal y del entorno, falta de agua potable, escasez de letrinas, 
etc.), de acuerdo a lo expresado en las encuestas de impacto, realizadas de manera individual.

- El impacto positivo alcanzado con la realización del proyecto en las comunidades de Santa Rosa,
San Rafael y Santa Lucía de Chuquipogyo, fue del $82 \%$ en los niños/as de entre 4 y 7 años de edad, y del $67 \%$ en los adultos/as, obteniendo una efectividad total del proyecto en un rango del $75 \%$, aproximadamente.

\section{Referencias bibliográficas}

1. ASAMBLEA CONSTITUYENTE (2008) Constitución del Ecuador 2008, Quito - Ecuador, 217 págs.

2. BOWER, B. y WERNER, D. (1993) Aprendiendo a Promover la Salud, Editado por la Fundación Hesperian, Palo Alto - California, Segunda Edición, 640 págs.

3. MINISTERIO DE SALUD PÚBLICA (2008) Indicadores Básicos de Salud Ecuador 2008, Editado por la Comisión de Indicadores Básicos de Salud y el equipo técnico, Quito - Ecuador, 17 págs.

4. WERNER, D., THUMAN, C. y MAXWELL, J. (2007) Donde no hay doctor, Editorial Pax - México, Distrito Federal - México, Segunda Edición, tercera actualización, 425 págs.

5. INSTITUTO NACIONAL DE ESTADíSTICAS Y CENSOS (INEC) (2006) La población indígena del Ecuador, Quito, Ecuador, Primera Edición, Talleres gráficos del Inec.

Páginas Web:

1. Disponible en Internet: $w w w$.triasandes.org/index.php?option=comcontent\&task=view\&id=18\&ltemid=136 (citado el 5 de noviembre del 2009; 12h54).

2. Disponible en Internet: http://www.flacso.org.ec/docs/lm_desarrollorural.pdf (citado el 7 de noviembre del 2009; 15h47).

3. Disponible en Internet: http://www.sld.cu/galerias/pdf/servicios/medicamentos/promocion_del_uso_racional_de_medicamentos_1.pdf (citado el 11 de noviembre del 2009; 10h12).

4. Disponible en Internet:

http://www.facmed.unam.mx/deptos/salud/censenanza/spi/fundamentos/navarro.pdf (citado el 21 de abril del 2010; 11h05).

5. Disponible en Internet:

http://apps.who.int/medicinedocs/en/d/Jh2973s/3.html (citado el 21 de abril del 2010; 12h01). 